\title{
Prospección de especies arbóreas para la fitorremediación de suelos contaminados por hidrocarburos, Amazonas, Perú
}

\author{
Prospection of tree species for the phytoremediation of \\ soils contaminated by hydrocarbons, Amazonas, Peru
}

Kerluin E. Yóplac ${ }^{1, \star}$, Oliver Tuesta ${ }^{1}$, Elí Pariente ${ }^{2}$ y Wagner Guzmán ${ }^{3}$

\begin{abstract}
Recibido: 06 febrero 2020 | Aceptado: 09 junio 2020 | Publicado en línea: 01 agosto 2020 Citación: Yóplac, KE; Tuesta, O; Pariente, E; Guzmán, W. 2020. Prospección de especies arbóreas para la fitorremediación de suelos contaminados por hidrocarburos, Amazonas, Perú. Revista Forestal del Perú 35(1): 31-41. DOI: http://dx.doi.org/10.21704/rfp.v35i1.1474
\end{abstract}

\begin{abstract}
Resumen
Esta investigación se realizó en el Anexo Inayo, distrito de Imaza, en el área contaminada por derrame de petróleo ocurrido el 25 de enero de 2016; tuvo como objetivo principal determinar qué especies nativas acumulan HTP (Hidrocarburos Totales del Petróleo) de forma natural; para ello se recolectaron e identificaron las especies que habitaban en el área afectada, estas fueron seleccionadas, procesadas y analizadas para cuantificar la concentración de HTP en las estructuras de la planta (hoja, raíz, tallo y fruto). Los resultados indican que las especies Piptocoma discolor, Theobroma cacao, Jacaranda copaía, Cedrela sp. y Schizolobium parahyba tienen la capacidad de bioacumular HTP en sus tejidos de forma natural; sobresaliendo la especie Piptocoma discolor que presentó mayor concentración en sus hojas con $28.306 \mathrm{mg} \cdot \mathrm{kg}^{-1}$, seguida por Theobroma cacao con 7.171 en raíz y $6.162 \mathrm{mg} . \mathrm{kg}^{-1}$ en tallo. Sin embargo, el análisis estadístico realizado muestra que ninguna especie tiene superioridad sobre la otra, respecto a la acumulación de este contaminante, por lo tanto se requieren de más estudios similares con estas especies para poder afirmar que son fitorremediadoras de suelos afectados por HTP.
\end{abstract}

Palabras clave: especies vegetales, hidrocarburos totales del petróleo (HTP), contaminación del petróleo

\footnotetext{
${ }^{1}$ Grupo de Investigación en Remediación de Suelos y Acuíferos (GIRSA), Facultad de Ingeniería Civil y Ambiental, Universidad Nacional Toribio Rodríguez de Mendoza, Calle Higos Urco № 342-350-356. Amazonas, Perú.

${ }^{2}$ Herbario de la Facultad de Ingeniería y Ciencias Agrarias de la Universidad Nacional Toribio Rodríguez de Mendoza de Amazonas, Perú.

${ }^{3}$ Superintendencia Nacional de Servicios de Saneamiento - SUNASS Amazonas, Perú.

* Autor de Correspondencia: kerluinyoplac.14@gmail.com
} 
Prospección de especies arbóreas para la fitorremediación de suelos contaminados por hidrocarburos, Amazonas, Perú

\begin{abstract}
This investigation was carried out in the Annex Inayo, district of Imaza, in the area contaminated by oil spill that occurred on January 25, 2016; Its main objective was to determine which native species accumulate Total Petroleum Hydrocarbons (TPH) in a natural way; For this, the species that inhabited the affected area were collected and identified, they were selected, processed and analyzed to quantify the concentration of TPH in the structures of the plant (leaf, root, stem). The results indicate that the species Piptocoma discolor, Theobroma cacao, Jacaranda copaía, Cedrela sp. and Schizolobium parahyba have the capacity to naturally bioaccumulate TPH in their tissues; standing out the species Piptocoma discolor that presented greater concentration in its leaves with $28.306 \mathrm{mg} . \mathrm{kg}^{-1}$, followed by Theobroma cacao with 7.171 in root and $6.162 \mathrm{mg} . \mathrm{kg}^{-1} \mathrm{in} \mathrm{stem}$. However, the statistical analysis carried out shows that no species has superiority over the other, regarding the accumulation of this pollutant, therefore more similar studies are required with these species to be able to affirm that they are phytoremediators of soils affected by TPH.
\end{abstract}

Key words: plant species, total petroleum hydrocarbons (TPH), petroleum pollution

\section{Introducción}

El suelo es un recurso natural indispensable para la vida del planeta, permite el establecimiento de actividades agrícolas, forestales y ganaderas (Acosta 2015, Rodríguez 2013); sin embargo, a nivel mundial, la gradual degradación del suelo se produce por contaminantes tóxicos orgánicos e inorgánicos, que pueden ser por causas naturales (erosión, filtraciones salinas) o antrópicas (derrames de petróleo, explotación de minerales) (Arthur et al. 2005, Buendía et al. 2014).

Los derrames de petróleo causan transformaciones en las propiedades físicas, químicas y biológicas del suelo (Cébron et al. 2011, Rivera-Cruz 2011), debido a la alta toxicidad del petróleo crudo, se ve afectada la densidad y la diversidad de las bacterias y hongos rizosféricos, disminuyendo la abundancia y riqueza de la fauna presente (Rivera-Cruz 2011, Uribe et al. 2010, Cutz-Pool et al. 2007). Consecuentemente, también se ven afectadas indirectamente las plantas, presentando alteraciones en su crecimiento y reproducción (Plaza et al. 2005, Rivera-Cruz et al. 2012). Algunas de las plantas presentes en suelos contaminados sobreviven valiéndose de mecanismos naturales y propios, como: la sorción, la liberación de exudados radicales y enzimas, y el incremento de la mineralización en la rizósfera (Schnoor y Lich 1995). De estos mecanismos la sorción es el principal fenómeno que influye en la biodisponibilidad de los compuestos orgánicos presentes en el suelo (Doménech y Peral 2006); y al mismo tiempo, este mecanismo depende de la etapa de crecimiento de la planta (Escalante y Gallegos 2005).

Hasta 2018 no existe un documento completo que integre, las especies vegetales que se adaptan a suelos contaminados con hidrocarburos; en Perú apenas existen esfuerzos en realizar estudios en esta línea de la fitorremediación. No obstante, las especies que sobreviven en suelos afectados por derrames de petróleo manifiestan una disminución en el crecimiento radicular y cobertura vegetal (Rivera et al. 2006). Por lo que en este trabajo se aborda la técnica de saneamiento biológico "fitorremediación" y enfocada en la identificación de especies arbóreas con capacidad de acumular HTP (Hidrocarburos Totales del Petróleo), con la finalidad de conocer qué especies vegetales tienen el potencial de fitorremediador de HTP, que ayuden a la conservación y manejo de los recursos naturales.

\section{Materiales y Métodos}

\section{Área de estudio}

La investigación se llevó a cabo en el Anexo Inayo, distrito de Imaza - Bagua, Amazonas Perú, por esta zona pasa el Oleoducto Norpe- 
ruano que transporta petróleo crudo desde la selva peruana hasta el terminal de Bayóvar ubicado en la costa norte del Perú. Se demarcó un área de estudio de 2.02 ha, ubicada en la margen izquierda de la Quebrada Inayo, afluente del Río Chiriaco, en las coordenadas geográficas $5^{\circ} 11^{\prime} 0.1^{\prime \prime}$ latitud sur $78^{\circ} 18^{\prime} 23^{\prime \prime}$ longitud oeste, en la zona de vida bosque muy húmedo Premontano Tropical. El 25 de enero de 2016, en el Km 440+781 del Tramo II del Oleoducto Norperuano (zona de estudio), ocurrió una falla del tubo que transportaba el petróleo, de 36 " de diámetro, produciendo una fuga de petróleo crudo, este discurrió e impregnó en el suelo, en las plantas de cacao, plátanos, y vegetación propia de la zona; además, fueron afectadas las áreas aledañas como el cauce y la franja marginal de la quebrada (nombre desconocido), quebrada Inayo y el río Chiriaco. Las áreas afectadas por el derrame de petróleo crudo (suelo, vegetación y agua superficial) fueron limpiadas y remediadas por Petroperú desde que ocurrió la emergencia ambiental.

El distrito de Imaza, se caracteriza por presentar temperatura promedio anual es $24.9^{\circ} \mathrm{C}$, con precipitaciones de hasta 2690.9 mm.año $^{-1}$; geomorfología de Montañas altas Calcáreas Mesozoicas con elevaciones por encima de los $1000 \mathrm{~m}$ de altitud, relieves de laderas moderadamente empinadas y alargadas, con cimas algo suaves y caprichosas.

\section{Selección del sitio de muestreo}

El sitio tenía que cumplir con los criterios seguidos por Ochoa-Gaona et al. (2011): a) que en el sitio haya ocurrido un derrame de petróleo, b) el año de ocurrido el derrame de petróleo y c) que los sitios hayan permanecido al menos seis meses del año sin inundación, y que estas áreas seleccionadas tuvieran comunidades vegetales presentes.

Características del sitio: a) en el sitio de muestreo ocurrió un derrame de petróleo en el Km 440+781 del Tramo II del Oleoducto Norperuano, b) sucedió el 25 de enero de 2016 ; c) para demostrar que el sitio no haya sido inundado se generó un mapa de pendientes el cual arrojó una pendiente moderada de $3 \%$ a $12 \%$, además, el área presentó vegetación arbórea representativa a una distancia máxima de 100 m desde el punto de fuga del petróleo crudo del oleoducto. Confirmando así que el sitio cumplía con los criterios de selección.

\section{Levantamiento de información}

Se realizó durante el mes abril de 2017, después de haber seleccionado el sitio de muestreo y delimitado el área de estudio. Se colectaron en paralelo muestras de suelo y especies vegetales (Figura 1).

\section{Colecta de muestras de suelo}

Se colectaron dos muestras de suelo (código M.S-P1 y M.S-P2) en el sitio de muestreo y una tercera muestra cerca al oleoducto (código M.S-OLE); se excavó en un área de $20 \times 20$ $\mathrm{cm}$ y a una profundidad de $30 \mathrm{~cm}$ empleando palana recta, se colocaron aproximadamente $500 \mathrm{~g}$ de suelo en bolsas herméticas y con sus respectivos códigos, posteriormente se enviaron al LABICER FC-UNI (Laboratorio de Investigación y Certificaciones de la Universidad Nacional de Ingeniería) para su análisis respectivo de HTP.

\section{Colecta de especies vegetales}

Sólo se colectaron muestras de los árboles adultos cuyo diámetro a la altura del pecho (DAP) $\geq 10 \mathrm{~cm}$, propuesto por Ochoa-Gaona et al. (2011), y estén localizadas dentro de las áreas afectadas por el derrame de petróleo. Se colectó una muestra de 100 g aproximadamente de cada una sus estructuras vegetales (raíz, tallo y hoja) por cada especie seleccionada, y solo se colecto el fruto de la única especie que presentó. Para la colecta de las muestras de hojas se empleó tijera telescópica y tijera de podar; las muestras de tallo se recolectaron a la altura del pecho $(1.30 \mathrm{~m})$, con dimensiones de $3 \mathrm{~cm}$ de ancho por $5 \mathrm{~cm}$ de largo a una profundidad de 3 a $4 \mathrm{~cm}$; las muestras de raíz se extrajeron excavando el suelo con un zapapico y se recortó $5 \mathrm{~cm}$ de largo con la ayuda de un machete. Cada muestra se colocó en papel absorbente (periódico) y se apiló en la prensa botánica junto a todas las muestras, asignando un código de registro diferente. Asimismo, 


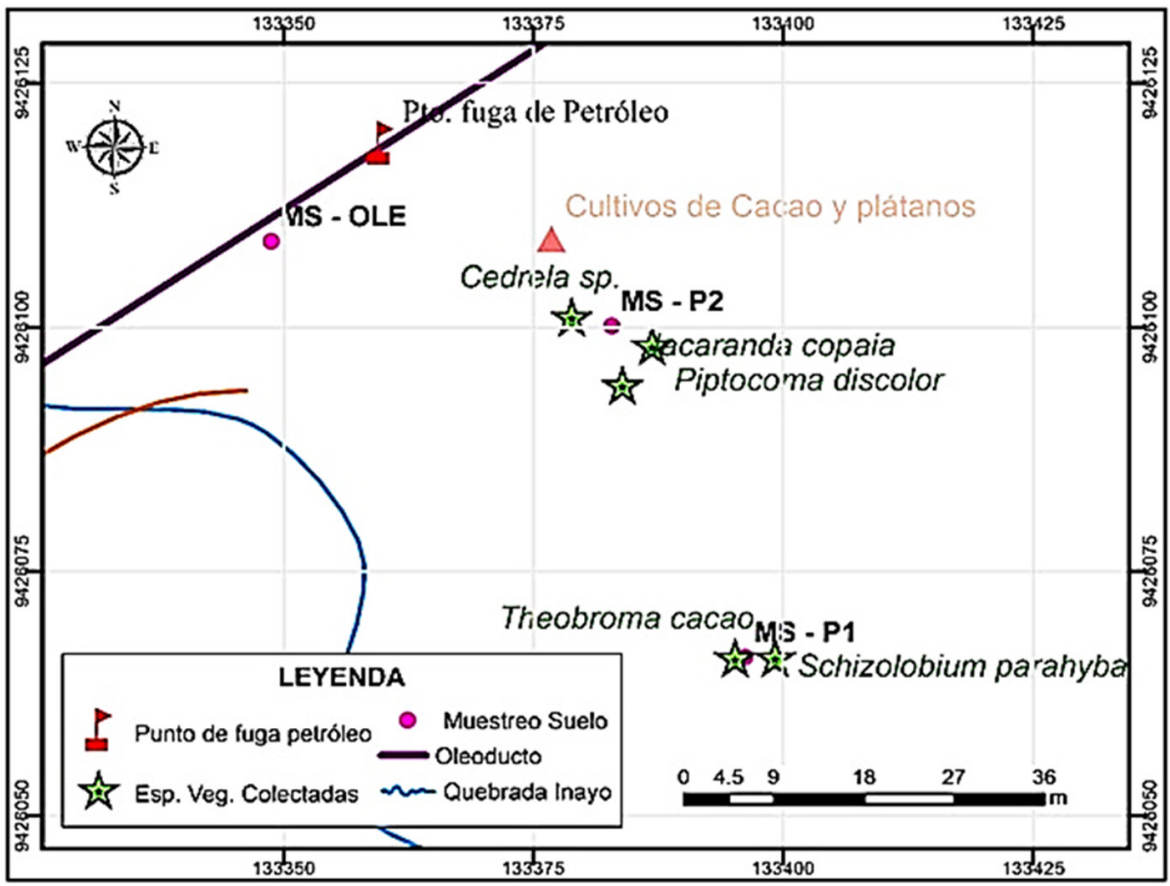

Figura 1. Mapa de muestras de suelo y especies vegetales colectadas.

se tomaron notas del nombre común y sus descripciones para cada una de ellas (circunferencia a la altura del pecho, tipo de ramificación, inflorescencia, flores, corteza interna y/o externa, altura, edad aproximada, frutos y usos), datos fundamentales para su identificación taxonómica.

Finalizada la colecta de muestras, se acondicionó las muestras y se cambió el papel periódico, doblando las ramitas y hojas de tal forma que estas se ajusten a la prensa botánica; se colocó alcohol de $96^{\circ}$ para preservar las muestras, luego se acondicionó en la prensa botánica y en bolsas de polietileno para su traslado y secado en el laboratorio LABISAG de la Universidad Nacional Toribio Rodríguez de Mendoza (UNTRM).

\section{Preparación de muestras para analizar HTP}

Se procedió al secado de las muestras de acuerdo a Bridson y Forman (1992), para ello se colocó la prensa con las muestras en una estufa a una temperatura de $90{ }^{\circ} \mathrm{C}$ durante tres
(03) días, se cambió a diario el papel periódico para el secado completo, evitar la contaminación y prevenir el sobre secado.

De las muestras secas, se seleccionaron y pesaron $15 \mathrm{~g}$ de cada una de las estructuras, de cada especie, y fueron enviadas al laboratorio LABICER de la Universidad Nacional de Ingeniería, para el análisis de la concentración de Hidrocarburos Totales del Petróleo.

\section{Identificación taxonómica de las especies}

Se realizó con ayuda bibliográfica especializada; cada especie arbórea fue identificada a nivel de familia, género, especie o morfoespecie, esto fue respaldado por especialistas del Herbario Forestal MOL de la Universidad Nacional Agraria La Molina (UNALM) y se realizó el depósito de los ejemplares en la misma institución.

\section{Análisis de datos}

Los datos fueron procesados, analizados, descritos, y representados en cuadros y figu- 
ras para su mejor interpretación y síntesis. A los datos obtenidos a nivel de laboratorio (concentraciones totales de HTP) se aplicó un tratamiento estadístico, empleando el software Statistix 8.0 para determinar si existe diferencia significativa en la acumulación de HTP, entre las cinco especies y sus estructuras vegetales. Primero se realizó un test de normalidad de Wilk y Shapiro, para determinar si los datos siguen una distribución normal, de ser el caso, se aplicaría una prueba paramétrica ANVA, de lo contrario se aplicaría la prueba no paramétrica de Friedman de doble vía para determinar: 1) diferencias significativas de la concentración entre especies y 2) diferencias significativas en la concentración de HTP entre las estructuras vegetales (hoja, raíz, tallo).

\section{Resultados}

\section{Levantamiento de información}

Los resultados de la concentración de Hidrocarburos Totales del Petróleo (HTP) en el suelo se presentan en el Cuadro 1. Tres muestras de suelo fueron analizadas del área de estudio, y se aprecia que las concentraciones de HTP son evidentes en el suelo (desde 1.733 a 6.860 mg. $\mathrm{kg}^{-1}$ ), lo cual fue un parámetro favorable para continuar con la investigación de encontrar especies prometedoras para la fitorremediación de suelos contaminados por hidrocarburos.

De las especies establecidas en el sitio, ninguna presentaba ningún tipo de deficiencia nutricional evidente o crecimiento anormal. Se encontraron y al mismo tiempo se determinaron cinco especies, consideradas silvestres (Cuadro 2). Estas especies son: Piptocoma discolor (Kunth) Pruski (Asteraceae), Theobroma cacao L. (Malvaceae), Jacaranda copaia (Aubl.) D.Don (Bignoniaceae), Cedrela sp. (Meliaceae) y Schizolobium parahyba (Vell.) S.F.Blake (Fabaceae), las cuales recibieron directamente hidrocarburos del petróleo. Estas cinco especies tenían un DAP mayor a $10 \mathrm{~cm}$ y su presencia era notoria en el área, con biomasa aprovechable para un mejor manejo en un sistema de saneamiento biológico del suelo, utilizando la técnica fitorremediación.
Concentración total de Hidrocarburos Totales del Petróleo en las estructuras vegetales por especie

Los resultados del análisis de concentración de Hidrocarburos Totales del Petróleo en las estructuras (hoja, raíz, tallo y fruto) de las especies colectadas que sobrevivieron al derrame de petróleo y con significativa presencia en el sitio (abundancia significativa), tenían mayor biomasa aprovechable para un mejor manejo en un sistema de fitorremediación, presentaron concentraciones de HTP, entre 5.231 a 28.306 mg.kg-1 en las hojas; de 2.115 a $7.171 \mathrm{mg} . \mathrm{kg}^{-1}$ en sus raíces y de 3.961 a 6.162 mg. $\mathrm{kg}^{-1}$ en sus tallos; no obstante, es importante mencionar que, de las cinco especies estudiadas, solo a una especie se le encontró fruto (Theobroma cacao) y este presentó una concentración de HTP (2.863 mg. $\mathrm{kg}^{-1}$ ) (Cuadro 3). Las concentraciones de HTP en las especies vegetales (Cuadro 3) son mayores a las concentraciones de HTP en el suelo (Cuadro 1).

De las especies analizadas, solo las especies Piptocoma discolor y Theobroma cacao presentan mayor acumulación de HTP; esta acumulación es notable en las hojas de las plantas, por lo tanto, estas podrían ser potenciales fitorremediadoras. Y cuando se realicen estudios más detallados y experimentales, y de acuerdo a los esfuerzos que estas expongan, podrían ser consideradas para su implementación dentro de un sistema de fitorremediación.

\section{Tratamiento estadístico de la concentración de Hidrocarburos Totales del Petróleo}

Se realizó la prueba de Wilk y Shapiro, para comprobar si los resultados de concentración de las estructuras de hoja, raíz y tallo, se ajustan a una distribución normal. La prueba arrojó el valor probabilístico $(p=0.0003)$ menor a $p=$ 0.01 , lo cual indica que, los datos no siguen una distribución normal (Figura 2). Dado que los datos no siguen una distribución normal y no se puede aplicar una prueba paramétrica, se aplicó la prueba de Friedman de doble vía para datos no paramétricos.

La prueba de Friedman arrojó el rango de concentración de HTP por especie, de- 
Prospección de especies arbóreas para la fitorremediación de suelos contaminados por hidrocarburos, Amazonas, Perú

\begin{tabular}{cccc}
\hline & \multicolumn{2}{c}{$\begin{array}{c}\text { Coordenadas UTM (WGS84) } \\
\text { Zona (17M) }\end{array}$} & Concentración (mg. \\
\cline { 2 - 3 } & Este & Norte & \multirow{2}{*}{ kg $^{-1}$ suelo seco) } \\
M.S - P1 & 0798644 & 9426388 & 6.860 \\
M.S - P2 & 0798631 & 9426422 & 1.733 \\
M.S - OLE & 0798597 & 9426431 & 3.476 \\
\hline
\end{tabular}

Cuadro 1. Concentración de Hidrocarburos Totales del Petróleo en muestras de suelo.

\begin{tabular}{lll}
\hline \multirow{2}{*}{ Familia } & \multicolumn{2}{c}{ Nombre } \\
\cline { 2 - 3 } Científico & Común \\
\hline Asteraceae & Piptocoma discolor (Kunth) Pruski & Yucate \\
Malvaceae & Theobroma cacao L. & Cacao \\
Bignoniaceae & Jacaranda copaia (Aubl.) D.Don & Vela \\
Meliaceae & Cedrela sp. & Cedro \\
Fabaceae & Schizolobium parahyba (Vell.) S.F.Blake & Plumilla \\
\hline
\end{tabular}

Cuadro 2. Listado de especies identificadas en el área de estudio.

\begin{tabular}{lcccc}
\hline Especie & Hoja & Raíz & Tallo & Fruto \\
\hline Piptocoma discolor & 28.306 & 6.421 & 3.961 & - \\
Theobroma cacao & 20.392 & 7.171 & 6.162 & 2.863 \\
Jacaranda copaia & 10.898 & 5.386 & 4.145 & - \\
Cedrela sp. & 10.729 & 4.474 & 5.294 & - \\
& & & & \\
Schizolobium parahyba & 5.231 & 2.115 & 4.173 & - \\
\hline
\end{tabular}

Cuadro 3. Concentración de Hidrocarburos Totales del Petróleo (mg.kg-1) obtenida por especies y estructura. 


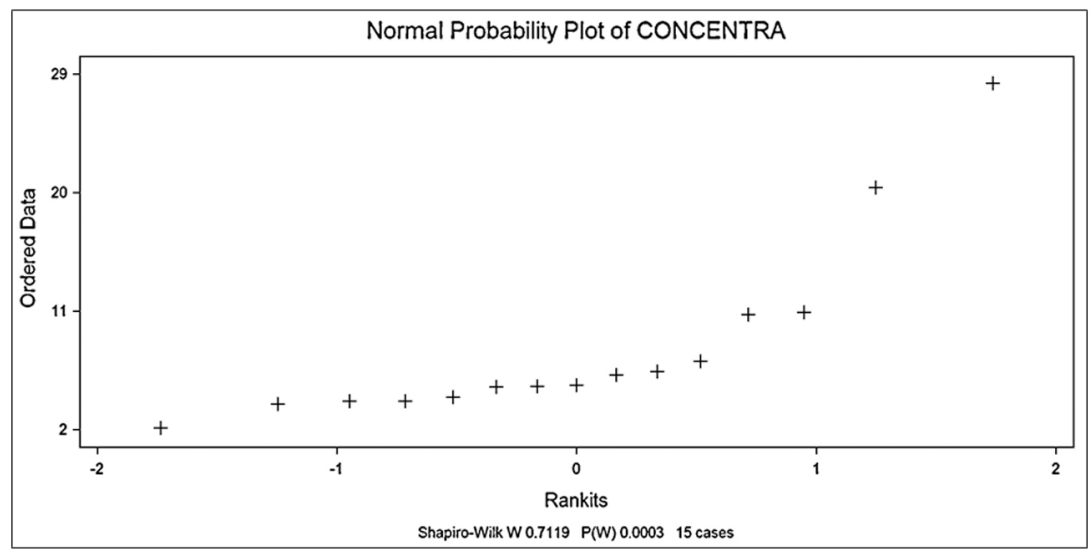

Figura 2. Prueba de Wilk y Shapiro para normalidad.

mostrando que no existe diferencia significativa entre las cinco especies silvestres estudiadas para la acumulación de Hidrocarburos Totales del Petróleo en sus estructuras (Cuadro 4).

Mientras que la concentración de HTP en las estructuras vegetales (hoja, raíz, tallo) de las cinco especies silvestres estudiadas, muestra que existe diferencia significativa para estas estructuras en la acumulación de HTP (Cuadro 5); siendo la hoja la estructura vegetal que mayor acumula HTP, seguidas por la raíz y finalmente el tallo.

\section{Discusión}

La concentración de HTP encontrada en el área evaluada no supera los Estándares de Calidad Ambiental (ECA) (Decreto supremo $\mathrm{N}^{\circ}$ 011-MINAM 2017) para suelos agrícolas según la normativa peruana (200 mg. $\mathrm{kg}^{-1}$ de HTP); estas bajas concentraciones de HTP en el suelo, se pueden explicar por la remediación del sitio y los diversos procesos de intemperización a los que son sometidos los contaminantes de forma natural (Al-Majed et al. 2012, Souza et al. 2014), además, los procesos de evaporación de las fracciones más volátiles de los hidrocarburos después de ocurrido el derrame ocasionados por la temperatura de la zona, dejando atrás compuestos de alto peso molecular que ofrecen resistencia a la degradación (Alagić et al. 2015). Errington et al. (2018) y Castellanos et al. (2015) afirman que la concentración de HTP por sí sola no indica toxicidad, asimismo, la sola presencia de los hidrocarburos del petróleo influye en las propiedades físicas y químicas de las capas del suelo directamente expuestas a los componentes tóxicos, afectando la microfauna del suelo y en las plantas disminuyen su presencia y la cobertura vegetal.

La disminución de la cobertura vegetal y la diversidad de especies arbóreas por la contaminación de hidrocarburos del petróleo se evidenciaron visualmente entre la vegetación del área del derrame y las áreas contiguas (suelos no afectados). Se seleccionaron cinco especies pertenecientes a las familias de las Asteraceae, Meliaceae, Malvaceae, Bignoniaceae y Fabaceae. Diferentes autores afirman que especies, pertenecientes a las mismas familias encontradas en nuestro estudio, contribuyen acelerando la degradación de los hidrocarburos del petróleo (Panchenko et al. 2018, Kaur et al. 2017, Arellano et al. 2017, Chan-Quijano et al. 2013, Ochoa-Gaona et al. 2011). Por lo tanto, se puede suponer que la presencia de las especies en el sitio pudo tener influencia en las bajas concentraciones de HTP en el suelo, debido a que especies de las mismas familias reportadas, son empleadas para degradar diversos hidrocarburos del petróleo. 


\begin{tabular}{lcc}
\hline Especie & Rango & Tamaño \\
\hline Piptocoma discolor & 3.33 & \\
Theobroma cacao & 4.67 & \\
Jacaranda copaia & 2.67 & 3 \\
Cedrela sp. & 2.67 & \\
Schizolobium parahyba & 1.67 \\
\hline
\end{tabular}

Nota: Friedman Statistic $=5.8667$

P-value, Chi-Squared Approximation $=0.2093$

Degrees of Freedom $=4$

Cuadro 4. ANOVA no paramétrico bidireccional de Friedman para "Concentración = Especie".

\begin{tabular}{lcc}
\hline Estructura & Rango & Tamaño \\
\hline Hoja & 3.00 & \\
Raíz & 1.60 & 5 \\
Tallo & 1.40 & \\
\hline
\end{tabular}

Nota: Friedman Statistic $=7.6000$

P-value, Chi-Squared Approximation $=0.0224$

Degrees of Freedom $=2$

Cuadro 5. ANOVA no paramétrico bidireccional de Friedman para "Concentración = Estructura".

También, las especies identificadas en el área de estudio demostraron características de especies fitorremediadoras, tales como, tolerar, resistir y sin daños evidentes por la presencia de los hidrocarburos del petróleo, que según Panchenko et al. (2018) y Schwitzguébel et al. (2011), las hacen especies potenciales e ideales para remediar áreas contaminadas con características similares al área de estudio y también, para poder evitar problemas de adaptación a suelos afectadas e invasión en los ecosistemas.
De las especies identificadas sobresalieron Piptocoma discolor con 28.306 mg. $\mathrm{kg}^{-1}$ de HTP en sus hojas, seguido por Theobroma cacao con concentraciones en raíz y tallo de 7.171 y 6.162 mg. $\mathrm{kg}^{-1}$ de HTP respectivamente; las especies analizadas difieren en su tolerancia y absorción de los contaminantes de hidrocarburos, tanto en concentración de HTP por especie y sus estructuras vegetales (raíz, tallo, hoja y fruto), características que permiten determinar las especies pueden ser hiperacumuladoras y/o acumuladoras de este contaminante (Barceló y Poschenrieder 2003, Rascio y Navari-Izzo 2011). Las especies vegetales identificadas se pueden considerar especies fitoextractoras de HTP por tener la mayor concentración del contaminante en la parte aérea, ya que reportaron mayores concentraciones de HTP en las hojas, características mencionadas por autores previos (Covarrubias y Peña 2017, Peralta-Pérez y Volke-Sepúlveda 2012).

Además, las concentraciones de HTP en suelos fueron menores en comparación con las concentraciones de HTP obtenidas en las cinco especies vegetales, indicando que las especies estudiadas son acumuladoras de este contaminante, porque tuvieron mayor concentración del contaminante en sus estructuras, comparada con la concentración del contaminante presente en el suelo donde estas están establecidas (Covarrubias y Peña 2017); por otro lado, la mayor concentración del contaminante en la parte aérea de la planta (tallo y hojas), en especial en las hojas, se debe a la mayor actividad metabólica y el almacenamiento de los nutrientes en estas estructuras (Raven et al. 1992).

Las especies Piptocoma discolor, Theobroma cacao y Jacaranda copaia son plantas fitoestabilizadoras porque tuvieron mayor concentración de HTP en su raíz, comparada con la concentración de HTP en el suelo, (Jara-Peña et al. 2014). Si bien durante la evaluación ninguna planta presento alguna anomalía, no significa necesariamente, que más adelante esta buena respuesta hacia las concentraciones de HTP en el suelo se mantenga. Por lo tanto se desconoce los resultados de concentración de HTP en las plantas, en el caso de que el análi- 
sis de suelo hubiera reportado concentraciones superiores, como por ejemplo por encima de los ECA para suelos (200 mg.kg-1 de HTP).

\section{Conclusiones}

Se identificaron cinco especies en el área afectada por el derrame de petróleo: Piptocoma discolor (Asteraceae), Theobroma cacao (Malvaceae), Jacaranda copaia (Bignoniaceae), Cedrela sp. (Meleaceae), y Schizolobium parahyba (Fabaceae). Sobresaliendo Piptocoma discolor con una concentración de $28.306 \mathrm{mg}$. $\mathrm{kg}^{-1}$ de HTP en sus hojas, seguido por Theobroma cacao con mayores concentraciones en raíz y tallo con 7.171 y 6.162 mg.kg-1 de HTP respectivamente.

El análisis estadístico realizado muestra que ninguna de las especies tiene ventaja sobre la otra; por lo tanto, no hay superioridad entre especies, respecto a la acumulación del contaminante.

De las cinco especies evaluadas, Theobroma cacao fue la única especie que presentó fruto (2.863 mg.kg-1 de HTP), colectada del área agrícola afectada por el derrame de petróleo. No obstante, se desconoce el riesgo a la salud humana en el caso de consumir sus frutos, debido a que la concentración de HTP no mide la toxicidad de los hidrocarburos presentes, además que la concentración de HTP en el suelo no supera los estándares de calidad ambiental (ECA) para suelos agrícolas.

Por lo tanto, el estudio se limita en afirmar que, las especies analizadas y encontradas sean fitorremediadoras, porque en el momento de la evaluación quizás las plantas no presentaron anomalías pero eso no quiere decir que si las presenten más adelante, hasta que estas sean sometidas a estudios más avanzados, para determinar la especie fitorremediadora que ayude a la conservación y manejo de los recursos naturales.

\section{Agradecimientos}

A la Universidad Nacional Toribio Rodríguez de Mendoza de Amazonas (UNTRM), por facilitarnos sus instalaciones. Al Dr. Ma- nuel Emilio Milla Pino por apoyarnos con el análisis estadístico de los datos de esta investigación. Al Sr. Linder Obet Arirua López por habernos guiado e informado, permitir el acceso a su predio para la toma de muestras de suelo y de las especies vegetales en las áreas afectadas por derrames de petróleo.

\section{Bibliografía}

Acosta, F. 2015. Guía para la planeación de proyectos de caracterización y remediación de suelos contaminados con hidrocarburos. Tesis Ing. Ciudad de México, México, UNAM. 131 p.

Alagić, SČ; Maluckov, BS; Radojičić, VB. 2015. How can plants manage polycyclic aromatic hydrocarbons? May these effects represent a useful tool for an effective soil remediation? A review. Clean Technologies and Environmental Policy 17(3):597-614.

Al-Majed, AA; Adebayo, AR; Hossain, ME. 2012. A sustainable approach to controlling oil spills. Journal of Environmental Management 113:213-227.

Arellano, P; Tansey, K; Baizter, H; Tellkamp, M. 2017. Plant Family-Specific Impacts of Petroleum Pollution on Biodiversity and Leaf Chlorophyll Content in the Amazon Rainforest of Ecuador. PLoS ONE 12(1):e0169867.

Arthur, E; Rice, P; Anderson, T; Baladi, S; Henderson, K; Coats, J. 2005. Phytoremediation-An Overview. Critical Reviews in Plant Sciences 24:109-122.

Barceló, J; Poschenrieder, C. 2003. Phytoremediation: Principles and perspectives. Contributions to Science 2:333-334.

Bridson, D; Forman, L. (1992). The Herbarium Handbook. The Board of Trustees of the Royal Botanic Gardens. 93 p.

Buendía, H; Cruz, F; Meza, C; Arévalo, J. 2014. Fitorremediación de suelos contaminados por hidrocarburos de petróleo. Alma máter 1(1):113-121.

Castellanos, ML; Isaza, RJ; Torres, JM. 2015. Evaluación de los hidrocarburos totales de 
petróleo (TPH) sobre suelos urbanos en Maicao, Colombia. Revista Colombiana de Química 44(3):11-17.

Cébron, A; Cortet, J; Criquet, S; Bíaz, A; Calver, V; Cauper, C; Leyval, C. 2011. Biological funtioning of PAH-polluted and thermal desortion-treated soils assessd by fauna and microbial indicators. Research in Microbiology 162(9):896-907.

Chan-Quijano, JG; Jarquín-Sánchez, A; Ochoa-Gaona, S; Bautista-Zúñiga, F; Martínez-Zurimendi, P; López-Chávez, MY. 2013. Especies vegetales útiles para fitorremediar suelos contaminados con hidrocarburos totales del petróleo: un apoyo para la restauración ecológica. Red Iberoamericana y del Caribe de Restauración Ecológica 7(3):7-14.

Covarrubias, SA; Peña, JJ. 2017. Contaminación ambiental por metales pesados en México: problemática y estrategias de fitorremediación. Revista Internacional Contaminación Ambiental 33:7-21.

Cutz-Pool, LQ; Palacios-Vargas, JG; Castaño-Meneses, G; García-Calderón, NE. 2007. Edaphic collembola from two agroecosytems with contrasting irrigation type in Hidalgo State, México. Aplied Soil Ecology 36(1):46-52.

Decreto supremo $\mathrm{N}^{\circ}$ 011-MINAM, 2017. Aprueban Estándares de Calidad Ambiental (ECA) para suelo. Diario oficial del Bicentenario El Peruano. Perú. 2 dic.

Doménech, X; Peral, J. 2006. Química ambiental de sistemas terrestres. Barcelona, España, Reverté. 256 p.

Errington, I; King, CK; Houlahan, S; George, SC; Michie, A; Hose, GC. 2018. The influence of vegetation and soil properties on springtail communities in a diesel-contaminated soil. Science of the Total Environment 619620:1098-1104.

Escalante, E; Gallegos, MM. 2005. Improvement of the hydrocarbon pytoremediation rate by "Cyperus laxus" Lam inoculated with a microbial Consortium in a model system. Chermosphere 59:405-413.
Jara-Peña, E; Gómez, J; Montoya, H; Chanco, M; Mariano, M; Cano, NC. 2014. Capacidad fitorremediadora de cinco especies altoandinas de suelos contaminados con metales pesados. Revista peruana de biología 21(2):145-154.

Kaur, N; Erickson, TE; Ball, AS; Ryan, MH. 2017. A review of germination and early growth as a proxy for plant fitness under petrogenic contamination - knowledge gaps and recommendations. Science of The Total Environment 603-604:728-744.

Ochoa-Gaona, S; Pérez, I; Frías, JA; Jarquín, A; Méndez, A. 2011. Estudio prospectivo de especies arbóreas promisorias para la fitorremediación de suelos contaminados por hidrocarburos. Secretaría de Recursos Naturales y Protección Ambiental y El Colegio de la Frontera Sur. Villahermosa, Tabasco, México. 144 p. Panchenko, L; Muratova, A; Dubrovskaya, E; Golubev, S; Turkovskaya, O. 2018. Dynamics of natural revegetation of hydrocarbon-contaminated soil and remediation potential of indigenous plant species in the steppe zone of the southern Volga Uplands. Environ Sci Pollut Res 25:3260-3274.

Peralta-Pérez, M; Volke-Sepúlveda, T. 2012. La defensa antioxidante en las plantas: una herramienta clave para la fitorremediación. Revista Mexicana de Ingeniería Química 11(1):75-88.

Plaza, G; Nalecz-Jawaki, G; Ugih, K; Brigmon, RL. 2005. The aplication of biossays as indicators of petrolum-contaminated soil reediation. Chermosphere 59(2):289-296.

Rascio, N; Navari-Izzo, F. 2011. Heavy metal hyperaccumulating plants: how and why do they do it? And what makes them so interesting? Plants Sci 180(2):169-181.

Raven, PH; Evert, RF; Eichhorn, SE. 1992. Biología de las plantas (4ta ed.). Barcelona, España: Reverté. 777 p.

Rivera, MC; Trujillo, A; Ferrera, R; Sánchez, P; Volke, V; Fernández, L; Rodríguez, R. 2006. Descontaminación con Benzo(a)pireno mediante microorganismos autóctonos y pasto alemán (Echinochleo polystachya [H.B.K.] 
Hitchc). Universidad y Ciencia Trópico Húmedo 22:1-12.

Rivera-Cruz, M. 2011. Bacterias y hongos en suelos contaminados con petróleo crudo en Tabasco. In Gamboa-Angulo, M; Rojas-Herrera, R. (eds.). .Recursos genéticos microbianos en la zona Golfo-sureste México México. p. 7787.

Rivera-Cruz, MC; Maldonado-Chávez, E; Trujillo-Narcía, A. 2012. Efects of crude oil on the growth of brachiaria mutica and Leucaena leucocephala and on soil and plant macronutrients. Tropical and Subtropical Agroecosystems 15(2):30-39.

Rodríguez, I. 2013. Reciclado en suelos de lodos de refinería: nuevas aproximaciones para la biodegradación de hidrocarburos mediante el manejo de enmiendas orgánicas. Tesis Doc. Ciudad de Murcia, España, Universidad de Murcia. 280 p.

Schnoor, Lj; Lich, AL. 1995. Phytorremediation of Organic Nutrient Contaminats. Environmental Science \& Technology 29(7):318-323.

Schwitzguébel, JP; Comino, E; Plata, N; Khalvati, M. 2011. Is phytoremediation a sustainable and reliable approach to clean-up contaminated water and soil in Alpine areas? Environmental Science and Pollution Research 18(6):842-856.

Souza, EC; Vessoni-Penna, TC; Oliveira, RP. 2014. Biosurfactant-enhanced hydrocarbon bioremediation: An overview. International Biodeterioration \& Biodegradation 89:88-94.

Uribe-Hernández, R; Juárez-Méndez, C; Oca, MA; Palacios-Vargas, JG; Cutz-Pool, L; Mejía-Recarmier, BE. 2010. Colémbolos (Hexápoda) como bioindicadores de la calidad de suelos contaminados con hidrocarburos en suelos en el sureste de México. Revista Mexicana de Biodiversidad 81(1):153-162. 